University of Nebraska - Lincoln

DigitalCommons@University of Nebraska - Lincoln

2006

\title{
Oxygen exposure promotes fuel diversity for Shewanella oneidensis microbial fuel cells
}

Justin C. Biffinger

US Naval Research Laboratory, justin.biffinger@nrl.navy.mil

Jacqueline N. Byrd

Naval Research Laboratory

Breanna L. Dudley

Naval Research Laboratory

Bradley R. Ringeisen

Naval Research Laboratory, ringeisen@nrl.navy.mil

Follow this and additional works at: https://digitalcommons.unl.edu/usnavyresearch

Part of the Operations Research, Systems Engineering and Industrial Engineering Commons

Biffinger, Justin C.; Byrd, Jacqueline N.; Dudley, Breanna L.; and Ringeisen, Bradley R., "Oxygen exposure promotes fuel diversity for Shewanella oneidensis microbial fuel cells" (2006). U.S. Navy Research. 13. https://digitalcommons.unl.edu/usnavyresearch/13

This Article is brought to you for free and open access by the U.S. Department of Defense at DigitalCommons@University of Nebraska - Lincoln. It has been accepted for inclusion in U.S. Navy Research by an authorized administrator of DigitalCommons@University of Nebraska - Lincoln. 


\title{
Oxygen exposure promotes fuel diversity for Shewanella oneidensis microbial fuel cells
}

\author{
Justin C. Biffinger, Jacqueline N. Byrd, Breanna L. Dudley, Bradley R. Ringeisen* \\ Chemistry Division, Naval Research Laboratory, 4555 Overlook Avenue, SW, Washington, DC 20375, United States \\ Received 18 May 2007; received in revised form 24 July 2007; accepted 31 August 2007 \\ Available online 6 September 2007
}

\begin{abstract}
Miniature microbial fuel cells (mini-MFCs) were used to monitor the current generated by Shewanella oneidensis DSP10 under both anaerobic and aerobic conditions when exposed to glucose as a potential electron donor. In addition to glucose, other carbon fuels including fructose, sucrose, acetate, and ascorbic acid were also tested. When the anolyte containing S. oneidensis was grown in the presence of oxygen, power densities of $270 \pm 10,350 \pm 20$, and $120 \pm 10 \mathrm{~W} / \mathrm{m}^{3}$ were recorded from the mini-MFC for glucose, fructose, and ascorbic acid electron donors, respectively, while sucrose and acetate produced no response. The power produced from glucose decreased considerably $\left(\leq 100 \mathrm{~W} / \mathrm{m}^{3}\right)$ if strictly anaerobic conditions were maintained. Based on the previously published proteomic and genomic literature on S. oneidensis, this reduction in power output is most likely due to the differential expression of proteins by these bacteria when grown under oxygen-rich or anoxic conditions. The power densities generated from the mini-MFC exposed to oxygen led to significant changes in current production over time with repeated feedings of these carbon nutrients. This work expands the breadth of potential electron donors for S. oneidensis MFCs and demonstrates the importance of studying microbial anolytes under diverse environmental conditions.
\end{abstract}

(C) 2007 Elsevier B.V. All rights reserved.

Keywords: Microbial fuel cell (MFC); Aerobic; Carbohydrate; Shewanella oneidensis

\section{Introduction}

Biological fuel cells offer a simple and direct method for converting biomass into electricity, which can be used to power autonomous aquatic sensors. Evidence already exists that energy can be generated from microbial fuel cells (MFCs) using naturally occurring microbial consortia and fuels found in sewage sludge (Park and Zeikus, 2003), waste water streams (Liu et al., 2004; Min and Logan, 2004; He et al., 2005), corn stover (Zuo et al., 2006), and even in isolated aquatic sediments (Tender et al., 2002). However, more information about energy production mechanisms and bacterial viability can be obtained when single strains are isolated from these consortia. The research performed with MFCs using a single species of metal reducing bacteria and simple carbon sources (such as acetate, formate, lactate) far outnumber those that utilize simple sugars, i.e. glucose (Chaudhuri and Lovley, 2003; Pham et al., 2003), directly. There are even

\footnotetext{
* Corresponding author. Tel.: +1 202767 0719; fax: +1 2024048119.

E-mail address: ringeisen@nrl.navy.mil (B.R. Ringeisen).
}

fewer pure culture MFC studies that use macromolecular carbohydrates directly (Niessen et al., 2004). Overall, a majority of MFCs that demonstrate the use of carbohydrates as electron donors involve either bacterial consortia or use the metabolic byproducts from bacterial fermentation to generate power (Shukla et al., 2004; Rabaey and Verstraete, 2005; Logan et al., 2006; Lovley, 2006).

Probing the adaptability of specific metal reducing bacterial species to complex fuels is imperative to enable and enhance energy harvesting from the environment by MFCs. The ability to couple the oxidation of organic and inorganic nutrients directly to metal reduction is widely distributed through the $\mathrm{Bac}$ teria and Archaea domains, with a majority of species identified in all five subdivisions $(\alpha, \beta, \gamma, \delta, \varepsilon)$ of the Proteobacteria family by $16 \mathrm{~S}$ RNA phylogenetic analysis (Lovley, 1993, 2000; Lovley et al., 2004). Dissimilatory metal reduction by microbes plays an essential part in the geochemical cycling of organic matter and metals in aquatic anoxic environments. By elucidating what conditions bacteria prefer specific nutrients to produce electricity and manipulating these bacteria or bacterial consortia to use these nutrients more efficiently, researchers 
can begin to reveal and enhance the complex energy scavenging processes that occur in consortia and/or sludge-based MFCs.

Under anaerobic conditions, one of the first isolated organisms to couple carbon utilization with iron reduction was from the Geobacteraceae family (Lovley, 1987). Members of this family are also the most abundant metal reducing microbes in the wide range of aquatic sub-sediments (Coates et al., 1996; Lovley et al., 1997, 2004). The study of Geobacter has enabled a more complete understanding of life in marine and freshwater sediments, but their oxygen sensitivity (Lin et al., 2004) and preferential growth on acetate makes them unlikely candidates for MFCs operating beyond obligate anaerobic or sedimentbased applications. The ability of $S$. oneidensis to grow under anaerobic and aerobic conditions, use a wide variety of electron acceptors, and secrete mediators to aid in electron transfer (potentially enhanced power densities), make $S$. oneidensis a provocative choice for a significantly wider variety of power applications in aerobic or microaerophilic environments. However, to date, only a small number of carbon containing electron donors (lactate, formate, pyruvate, amino acids, hydrogen) have supported metal reduction by $S$. oneidensis under anaerobic conditions (Nealson et al., 2002). In aerobic environments, these electron donor limitations are not as pronounced. For example, a recent paper describes chitin as a nutrient that supports growth and energy production by Shewanella sp. under aerobic conditions (Yang et al., 2006).

Through careful design modifications to a MFC, a wider variety of carbon fuels and bacterial species could be monitored for power output, while in the presence of air as opposed to maintaining strictly anaerobic conditions. For example, maximizing the electrode surface area-to-chamber volume ratio will decrease the void space in the anode chamber, thereby increasing the number of electrons harvested by the anode. This approach would result in an increase in power output while maintaining the anolyte culture in air. The mini-MFC fabricated by Ringeisen and co-workers is one such device that specifically addresses the issue of electrode surface area-to-volume ratio. The mini-MFC design has enabled the oxygen tolerance of microbes to be studied with regards to power output. Specifically, the demonstration of power output from Shewanella sp. under various levels of oxygen exposure has been reported (Ringeisen et al., 2007, 2006). Using a three-dimensional (3D) low density graphite felt electrode within the small anode chamber $(1.2 \mathrm{~mL})$ creates an environment where oxygen was scavenged by $S$. oneidensis in the anode chamber with only a $40 \%$ drop in power output (Ringeisen et al., 2007). Remarkably, this power production was recorded when the inlet anolyte oxygen concentration was measured at $9 \mathrm{ppm}$ (air-saturated) while the effluent concentration was reduced to $1 \mathrm{ppm}$.

In addition to the large power densities and small working volumes, the mini-MFC also provides a quick way to analyze the small power differences generated between different bacterial species under a variety of conditions. Instead of comprising the bulk of the anodic operating volume with the bacterial culture (batch reactor), the bulk of the anolyte within the mini-MFC is maintained external to the anode work- ing volume (Supplementary Fig. S1). This creates two distinct environments: one with reduced oxygen that enables power production (anode chamber), while the other environment can be exposed to varying degrees of aeration and growth conditions (culture flask). This design consideration is also the reason why power can be produced in the presence of oxygen and is the feature of the mini-MFC that is unique from standard two chamber laboratory MFCs.

Here, we report power output from the mini-MFC with pure culture $S$. oneidensis DSP10 utilizing glucose under anaerobic and aerobic conditions. The change in power response after subsequent glucose additions indicates that aerobic $S$. oneidensis was adapting to the presence of glucose with time. We find that aerobic growth and the mini-MFC design improves the capability of $S$. oneidensis to respond to complex carbon nutrient sources while maintaining power output. Also, this work demonstrates that $S$. oneidensis grown with glucose in the presence of oxygen generates more power than under strictly anaerobic conditions where the elimination of oxygen should typically increase the fuel cell efficiency and increase power output. Therefore, an increase in power under oxygen exposure is an indication that aerobic $S$. oneidensis can effectively utilize complex carbon sources as electron donors in MFCs.

\section{Material and methods}

Aliquots of the electron donors used for this work were added from standard stock solutions (1 M D-glucose, $1 \mathrm{M} \mathrm{D-}$ fructose, $0.5 \mathrm{M}$ sucrose, $1.95 \mathrm{M}$ sodium acetate, $0.5 \mathrm{M}$ ascorbic acid) stored at $5^{\circ} \mathrm{C}$ in $100 \mathrm{mM}$ phosphate buffer ( $\mathrm{pH}$ 7.2). The solvent for the solutions was Millipore $18 \mathrm{M} \Omega$ water.

\subsection{Cell culture conditions}

For aerobic experiments, the DSP10 strain of the S. oneidensis (obtained from Dr. Kenneth Nealson (University of Southern California, Los Angeles, CA) on an agar slant) was used. Aerobic mini-MFC experiments used $S$. oneidensis DSP10 that were sub-cultured aerobically in $50 \mathrm{~mL}$ of Luria-Bertani (LB) broth at $25^{\circ} \mathrm{C}, 100 \mathrm{rpm}$, and were started initially from frozen stock cultures (equal amount of DSP10 culture and sterile glycerol at $-80^{\circ} \mathrm{C}$ ). Anaerobic mini-MFC experiments used cultures that were created by inoculating $50 \mathrm{~mL}$ of degassed (nitrogen purge $5 \mathrm{~min}$ ) LB with $5 \mathrm{~mL}$ of a $1 \pm 2 \times 10^{7}$ cells $/ \mathrm{mL}$ aerobic culture of $S$. oneidensis with and without $10 \mathrm{mM} \mathrm{Fe(III)} \mathrm{citrate} \mathrm{as} \mathrm{an}$ electron acceptor. This culture was placed in an anaerobic glove box at $28^{\circ} \mathrm{C}$ with agitation at $150 \mathrm{rpm}$ for 4 days prior to starting the fuel cell experiment.

\subsection{Fuel cell assembly and operation of the mini-MFC}

The general dimensions and setup for the mini-MFC apparatus were described previously (Ringeisen et al., 2006) and are depicted in the Supplementary Fig. S1. The electrodes used within the fuel cell chambers were low-density graphite felt (0.13 g, Electrosynthesis company, Lancaster, NY; $\left.0.47 \mathrm{~m}^{2} / \mathrm{g}\right)$ 
and were connected with titanium wires to an external load. The volume of each electrode chamber was $1.2 \mathrm{~mL}$, and the anode and cathode chambers were separated by Nafion ${ }^{\circledR}-117$ (The Fuel Cell Store, membrane pre-treated with for $1 \mathrm{~h}$ each in hot de-ionized (DI) water, $3 \%$ hydrogen peroxide, $1 \mathrm{M}$ sulfuric acid, and DI water again). The anolyte and catholyte were passed through the chambers at a flow rate of $1-2 \mathrm{~mL} / \mathrm{min}$ using a peristaltic pump. To assure that the power response was not due to residual LB, the power was allowed to stabilize and then gradually decline to a stabilized minimum before any electron donor was added. The nutrients tested were added as aliquots from stock solutions (stored at $4{ }^{\circ} \mathrm{C}$ ) to the anolyte culture flask attached to the fuel cell. The catholyte for each fuel cell was a $50 \mathrm{mM}$ potassium ferricyanide solution in $100 \mathrm{mM}$ phosphate buffer ( $\mathrm{pH}$ 7.2) using uncoated GF electrodes. All fuel cells were run between $21 \pm 1{ }^{\circ} \mathrm{C}$. For aerobic mini-MFC experiments, the unstirred aerobic $S$. oneidensis culture was exposed to air in the reservoir flask. Dissolved oxygen measurements (ISO2 probe, WPI, Inc., Tampa, FL) for both influent and effluent lines indicated that the $S$. oneidensis culture maintained low dissolved $\mathrm{O}_{2}$ levels $(0.2 \pm 0.2 \mathrm{ppm})$ within the fuel cell (Ringeisen et al., 2006) due to respiration. Anaerobic mini-MFC experiments were performed by bubbling sterile nitrogen gas $(0.45 \mu \mathrm{m}$ filtered) through the anaerobic culture in the reservoir flask (dissolved $\mathrm{O}_{2}$ below detectable limit).

\subsection{Cell counts and culture integrity}

S. oneidensis DSP10 cultures were monitored for contaminants and viability by periodic spread plating of the anolyte after serial dilution on LB/agar throughout fuel cell operation. The dilutions were performed in $9 \%$ sodium chloride solutions. The inverted plates were grown aerobically at $30^{\circ} \mathrm{C}$.

\subsection{Data acquisition}

The voltage across a load was measured by a personal data acquisition device (IO tech, personal daq/54) every $2 \mathrm{~min}$. The measured voltage was converted to current through Ohm's law (Voltage $=$ Current $\times$ Resistance $)$ and to power using $($ Power $=$ Current $\times$ Voltage $)($ Logan et al., 2006). Voltage measurements for power curves were allowed to reach steady state $(\sim 5 \mathrm{~min})$. Coulombic efficiency $(E)$ was calculated from published protocols using the formula $E(\%)=\left(C_{\mathrm{p}} / C_{\mathrm{T}}\right) \times 100$, where $C_{\mathrm{p}}$ is the total coulombs calculated by integrating the current over time and $C_{\mathrm{T}}$ are the theoretical amount of coulombs, calculated as,

$C_{\mathrm{T}}=b F\left(\mathrm{~mol}_{\text {nutrient }}\right)$

where $F$ is Faraday's constant, $b$ are the number of electrons potentially transferred from the nutrient, and $\mathrm{mol}_{\text {nutrient }}$ are the moles of the nutrient consumed by the bacteria. The number of potential electrons used for glucose, fructose, lactate, and ascorbic acid were 24, 24, 12, and 2, respectively. Power measurements were performed in triplicate and the average value was used.

\section{Results}

\subsection{Metabolism of glucose by S. oneidensis DSP10 from anaerobic to aerobic environments}

The power curves from the mini-MFCs containing $S$. oneidensis DSP10 using glucose under aerobic (culture exposed to air) and anaerobic (culture bubbled with nitrogen) conditions are presented in Fig. 1. In general, to assure that the power response was not due to residual LB, the power output was allowed to stabilize and eventually decrease before any nutrient was added. Both the maximum power and short circuit current generated under aerobic conditions ( $320 \mu \mathrm{W}, 1.6 \mathrm{~mA})$ were approximately three times greater than under anaerobic conditions $(110 \mu \mathrm{W}$, $0.48 \mathrm{~mA})$. A slightly lower open circuit voltage $(0.7 \mathrm{~V}$ versus $0.8 \mathrm{~V}$ ) was observed for the aerobic culture. This decrease is most likely due to the presence of trace amounts of oxygen in the anode chamber that would reduce the overall potential difference between the anode and cathode compartments (Fig. 1, inset).

Previous results when using lactate as an electron donor showed a decrease in power when the anolyte was exposed to oxygen (Ringeisen et al., 2007). For the glucose results, we find the opposite trend where purging oxygen from the anolyte decreased the power output. These results show that glucose is not used effectively under strictly anaerobic conditions for power generation, while a $S$. oneidensis culture exposed to air increases the power output dramatically.

A possible alternative explanation for the large differences in the power output by anaerobic and aerobic cultures could be either due to a lower concentration of $S$. oneidensis when grown anaerobically or a contaminant species co-existing in the anolyte that ferments glucose to functional by-products for $S$. oneidensis metabolism. We addressed these issues by following the cell counts and purity of the cultures through periodic serial dilutions using streak plates of the anolyte while operating the mini-MFC. The power versus time data is shown in Fig. 2 for a mini-MFC operated under aerobic conditions. The spikes in power occur after five sequential additions of $10 \mathrm{mM}$ glucose and the block arrows indicate when aliquots were withdrawn from the anolyte for streak plate analysis. Overall, the concentration

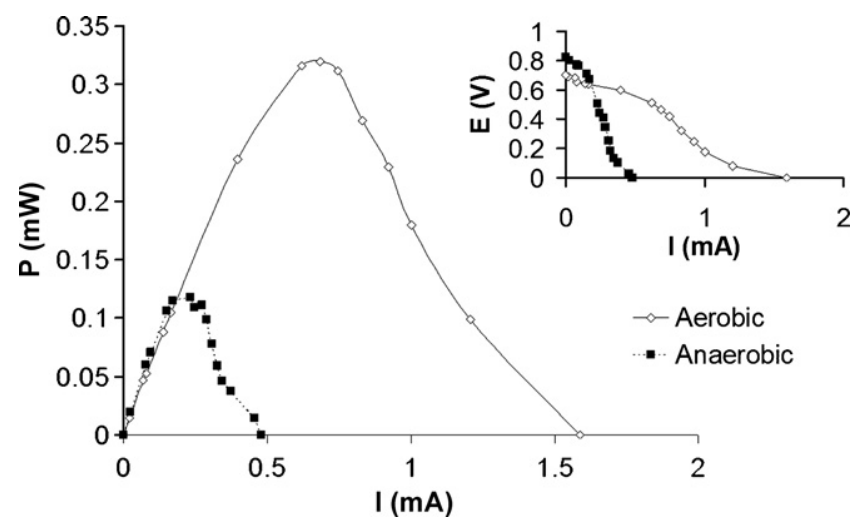

Fig. 1. Power vs. current for S. oneidensis DSP10 using glucose under anaerobic and aerobic conditions (inset: polarization plot for the response from glucose). 


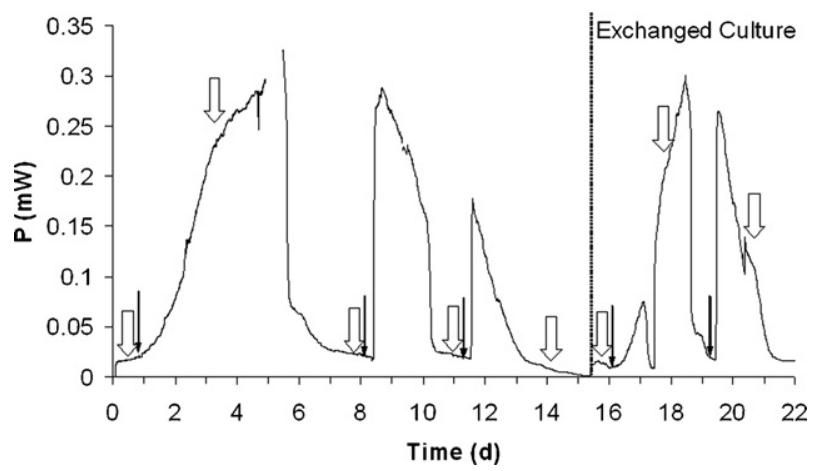

Fig. 2. Power production with time for a mini-MFC using aerobic $S$. oneidensis DSP10 cultures with periodic glucose additions $(\rightarrow)$ and block arrows signifying when serial dilution aliquots were withdrawn (external load: $560 \Omega$ ).

of S. oneidensis remained nearly constant $\left(3 \pm 1 \times 10^{7}\right.$ cells $\left./ \mathrm{mL}\right)$ over the first 11 days of mini-MFC operation for both the anaerobic (data not shown) and aerobic cultures. Thus, the difference in power when using glucose under anaerobic versus aerobic conditions was not due to dramatically lower cell concentrations. Periodic serial dilution of the anolyte also aided in determining whether there were other contaminant bacterial species present in the anolyte. Considering the liquid base of the anolyte was LB exposed to air, plating on aerobic LB/agar will support growth of both the $S$. oneidensis and any contaminant that could also be growing in the culture. No such bacterial contaminant was observed while operating the fuel cell over the first 2 weeks under all conditions. Since there were consistent cell counts and no competing contaminant bacteria in the anolyte, we conclude that the power spikes observed under aerobic conditions after repeated additions of glucose (Fig. 2) were solely due to the metabolism of glucose by $S$. oneidensis.

The variable power response with time of the mini-MFC throughout the experiment provided some insight as to why the addition of glucose generated power under these conditions. Each aerobic mini-MFC used to monitor the power produced from glucose showed a consistent and slow (4-6 day) response to the initial glucose addition, followed by a significantly faster $(1 \mathrm{~h})$ maximum power response after subsequent additions (black arrows indicate glucose addition, Fig. 2). This behavior is unique, as previous mini-MFC experiments at the same flow rate using sodium lactate as the electron donor show an immediate response $(<1 \mathrm{~h})$ after every addition (Ringeisen et al., 2006).

A similar slow initial response to glucose was observed with MFCs containing Proteus vulgaris (Kim et al., 2000) and Gluconobacter oxydans (Lee et al., 2002). This slow response was attributed to either an increase in concentration of spontaneous mutants expressing proteins for glucose metabolism or directed evolution. The evolution of bacteria in these experiments is dependent on both how they are grown and the electron donor. The data shown in this manuscript (Fig. 2) is consistent with these conclusions as well. The immediate power produced after the first addition of glucose is most likely from the S. oneidensis initially acclimated to metabolize glucose. While the second and third glucose additions result in sharper power rises due to pre-conditioning of the culture and possible increased concentration of $S$. oneidensis mutants able to efficiently metabolize glucose. The gradual decline of power production at the end of 2 weeks was due to a decrease in the overall cell concentration of $S$. oneidensis in culture. This decrease in power production was also observed by Kim and co-workers with $S$. oneidensis after successive additions of lactate over time but no definitive conclusion was provided (Kim et al., 2002). When the original $S$. oneidensis culture was exchanged with a new (3 days old) aerobic culture (Fig. 2, data after 15 days), another gradual response (days 16-21) was observed of the same magnitude as the initial response observed on days 1-6. This response time was slightly shorter than for the original culture (3 days rather than 6 days), suggesting that a partial biofilm of glucose-acclimated S. oneidensis may have been formed on the anode.

\subsection{Alternative carbon nutrients for S. oneidensis DSP10}

This is the first paper to demonstrate power production from S. oneidensis with glucose as the only available electron donor. Additional experiments were performed to probe other nontraditional electron donors, that is, nutrients not capable of solely supporting the growth of $S$. oneidensis under strictly anaerobic conditions, but could potentially be utilized under aerobic conditions. A range of simple (acetate) to complex (sucrose, fructose, ascorbic acid) carbon nutrients or cofactors involved in distinct biochemical pathways in prokaryotic metabolism were examined (Table 1). The power generated over time by aerobic $S$. oneidensis for fructose as the electron donor is shown in Fig. 3. The slow response of S. oneidensis to fructose was consistent with the slow initial response observed during the microaerophilic glucose experiments and was excluded from Fig. 3. After 5 days, all subsequent additions of fructose resulted in an immediate response $(0.4 \mathrm{~mW})$, consistent with the gradual acclimation of $S$. oneidensis to effectively utilize fructose. Ascorbic acid also generated a power response from $S$. oneidensis, though it was significantly lower than all the other current generating nutrients tested $(0.1 \mathrm{~mW}$, Fig. $4 \mathrm{~A})$.

Sucrose did not generate any power above the background response after either addition to the anolyte over the course of 6 days (Supplementary Fig. S2). To confirm that the S. oneidensis culture was still viable, $10 \mathrm{mM}$ sodium lactate was then added,

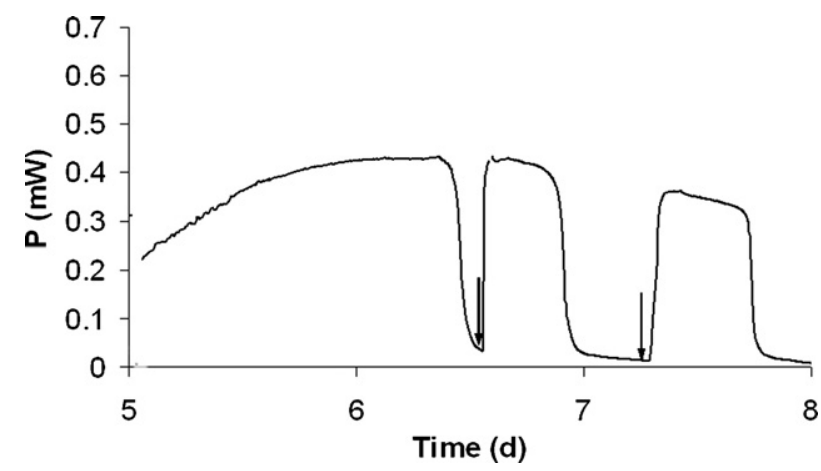

Fig. 3. The power generated from a S. oneidensis DSP10 aerobic culture with periodic $10 \mathrm{mM}$ fructose additions (external load: $680 \Omega$ ). 
Table 1

Summary of MFC results using S. oneidensis DSP10 under an aerobic atmosphere in the mini-MFC

\begin{tabular}{|c|c|c|c|c|c|c|}
\hline Nutrients & $E_{\mathrm{oc}}(\mathrm{V})$ & $I_{\mathrm{sc}}(\mathrm{mA})$ & Coulombic efficiency (\%) & Theoretical end product & Power density $\left(\mathrm{W} / \mathrm{m}^{3}\right)$ & Reference \\
\hline Acetate & 0.51 & - & - & - & - & This work \\
\hline Lactate & 0.75 & 2.0 & $8 \pm 1$ & $\mathrm{CO}_{2}$ & $500 \pm 30$ & Ringeisen et al. (2006) \\
\hline Glucose & 0.84 & 1.6 & $30 \pm 4$ & $\mathrm{CO}_{2}$ & $270 \pm 10$ & This work \\
\hline Fructose & 0.69 & 1.3 & $10 \pm 1$ & $\mathrm{CO}_{2}$ & $350 \pm 20$ & This work \\
\hline Sucrose & 0.52 & - & - & - & - & This work \\
\hline Ascorbic acid & 0.73 & 2.2 & $9 \pm 1$ & Dehydroascorbic acid & $120 \pm 10$ & This work \\
\hline
\end{tabular}

resulting in a power consistent with previous work (Ringeisen et al., 2006). Acetate was also studied under these same conditions with no current generated above the background after several additions (Supplementary Fig. S3). Conditions that resulted in no power output held an open circuit voltage (OCV) of $0.5 \mathrm{~V}$ while an OCV of 0.6-0.8 V was observed for glucose, fructose, and ascorbic acid. Using the standard reduction potential for ferricyanide ( $E^{\circ}=430 \mathrm{mV}$ versus NHE (Roncel et al., 2001)) the resting potential for $S$. oneidensis was $-70 \pm 1 \mathrm{mV}$ versus $\mathrm{NHE}$ and as high as $-370 \pm 3 \mathrm{mV}$ versus NHE when current was generated. Previously reported electrochemical studies also resulted in standard redox potentials of comparable magnitude $(-100 \mathrm{mV}$ versus NHE) for Shewanella and lactate (Kim et al., 1999).

The amount of current generated versus time from a single molecule of glucose, ascorbic acid, and fructose, was used to calculate the Coulombic efficiencies shown in Fig. 4A (Table 1). For each molecule used, the maximum number of potential elec-
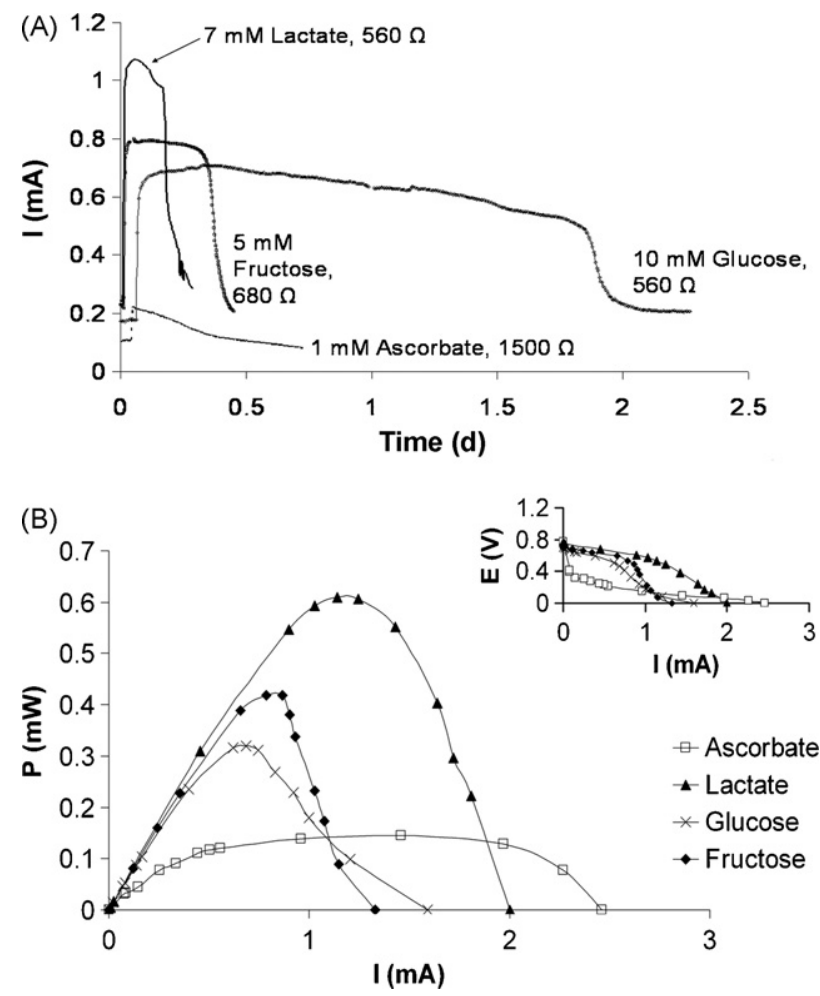

Fig. 4. (A) Current vs. time plot for lactate, glucose, fructose, and ascorbate with $S$. oneidensis DSP10 exposed to oxygen and (B) power vs. current plots for each of the before mentioned nutrients (inset: voltage vs. current data for the same nutrients). trons was applied, making these efficiency values a lower limit. This assumption is reasonable considering under aerobic conditions Shewanella saccharophilia only generated $\mathrm{CO}_{2}$ when metabolizing glucose (Coates et al., 1998). The addition of glucose to $S$. oneidensis produced the highest Coulombic efficiency $(30 \pm 4 \%)$, while fructose, ascorbic acid, and lactate generated comparable, lower efficiencies (8-10\%). However, from the calculated power densities, lactate and fructose generated the highest power of all the electron donors tested for this work (Fig. 4B). These observations are consistent with a longer but lower power response with glucose, while lactate and fructose generated a short but higher power response (Fig. 4A). Varying external loads were used for efficiency measurements (Fig. 4B) because the resistor that generated the peak power was different between nutrients. The variable resistance between nutrients was most likely due to either how effectively the bacteria were generating current or changes in the internal resistance of the MFC.

Under aerobic conditions, S. oneidensis consumed glucose, fructose, and ascorbic acid to generate current from the miniMFC, while there was no response for acetate and sucrose. All of these carbon nutrients have been previously described as unusable for anaerobic growth on $\mathrm{Fe}(\mathrm{III})$ in S. oneidensis culture (Nealson and Saffarini, 1994). These data highlight the importance of oxygen exposure and monitoring growth conditions to help diversify MFCs and enable higher order carbohydrates to be utilized as electron donors.

\section{Discussion}

A fairly complete analysis of protein expression and gene transcription of $S$. oneidensis MR-1 under anaerobic and aerobic conditions has been reported recently (Saffarini et al., 1994; Scott and Nealson, 1994; Beliaev et al., 2002, 2005; Heidelberg et al., 2002; Giometti et al., 2003; Kolker et al., 2005). It is clear from these studies that $S$. oneidensis possess pathways to metabolize carbohydrates and amino acids. However, the expression of certain key proteins involved in the tricarboxylic acid (TCA), pentose phosphate, and Entner-Doudoroff pathways are low under anaerobic conditions; conditions that nearly all previous MFC experiments are performed under to limit electron scavenging by dissolved oxygen in the anode chamber. Under aerobic conditions, these proteins are expressed at higher levels (Beliaev et al., 2002, 2005; Kolker et al., 2005), indicating that $S$. oneidensis should have all the biochemical tools to utilize glucose and other more complex sugars when maintained under aerobic conditions. 
These data indicate that MFCs utilizing aerobic cultures of S. oneidensis could be capable of generating current from a much wider variety of nutrients than those using anaerobic cultures. It is promising that just a simple change in culturing conditions and the mini-MFC design resulted in current generation from a nutrient previously considered unusable. However, unlike the Geobacter sp., our experiments show that S. oneidensis still does not metabolize acetate. Acetate is the end product of several metabolic pathways for higher order carbon sources (including the Entner-Doudoroff pathway for glucose metabolism) and should be considered by S. oneidensis more as a waste product from metabolism rather than a nutrient for growth. Even though data presented here show that $S$. oneidensis has a preference for lactate under all conditions, acetate remains unusable by this microbe for current production.

The other nutrient that did not generate current from $S$. oneidensis was sucrose. Sucrose is a dimer of both glucose and fructose components and is involved in the storage of these simple sugars within energy production pathways. Sucrose was the most complex carbon source studied and would be a challenge for $S$. oneidensis to utilize considering no proteins for sucrose metabolism have been annotated or identified. However, the metabolism of sucrose could be partitioned for membrane and cell viability instead of for the externalization of electrons through either mediators or directly to the electrode surface.

The ability for a microbe to live in an oxygen-rich environment requires methods to resist damage by free radical peroxides resulting from the incomplete reduction of oxygen. Recent proteomic and genomic work with $S$. oneidensis has shown that several proteins with antioxidant properties such as superoxide dismutase or other toxic radical scavenging systems are expressed under both high and low concentrations of oxygen (Giometti et al., 2003; Beliaev et al., 2005; Kolker et al., 2005). Ascorbate is a glucose analog and a cofactor in numerous biological processes including antioxidant defense at several levels in eukaryotes and even mediating electron transfer with ascorbatedependent peroxidases (Halliwell, 2001). Considering the large number of iron and copper-containing enzymes expressed by metal reducing bacteria, ascorbate was regarded as a unique molecule that should be used by a facultative anaerobe grown with oxygen as the terminal electron acceptor. The short circuit current for ascorbate under microaerophilic conditions was comparable to using lactate as a nutrient, but the maximum power of $120 \pm 10 \mu \mathrm{W}$ produced by ascorbate was substantially lower than the maximum power generated by lactate and roughly half that produced after fructose and glucose additions (Table 1).

There are several potential explanations for the high current (yet low power) generated from ascorbate. In general, short circuit current is a measure of the maximum current the fuel cell can produce and is measured under diffusion limited conditions; hence, these are the experimental conditions where the most electrons from the bacteria are required. To ensure that the changes in power were not from the cathode, all experiments were run with $50 \mathrm{mM}$ ferricyanide as the catholyte with a bare graphite felt electrode. Large changes in solution conductivity that would reduce IR drop are also not consistent with these results, considering the elevated power for glucose and low power for ascorbate. The sharp drop in the polarization curve for ascorbate at low current is different from the data for the other electron donors and may be indicative of slower kinetics for this molecule (Fig. 4B, inset). Thus, one possible explanation for the low power observed for ascorbate is the inability for $S$. oneidensis to effectively metabolize this molecule, resulting in slow kinetics and immediate loss of potential under high external loads.

MFCs containing microbial consortia are currently the most efficient at converting simple (and in come cases complex) carbohydrates directly into current. However, determining what component of a microbial consortium is producing the power and how the consortia of microbes are using the nutrients is a complicated challenge. Working with pure cultures of bacteria might not produce power maxima, but it does provide insight into mechanisms that would otherwise be masked by the presence of other bacteria. Clearly, the differential expression of proteins under aerobic conditions negates the limitations of $S$. oneidensis under anaerobic conditions (limited diversity of electron donors). The experiments presented here may lead to new directions in MFC research, specifically investigations that utilize pure cultures and non-traditional environments to elucidate the complex mechanisms that occur in the anolyte for power production.

\section{Conclusions}

Using the mini-MFC design, fuels such as glucose and fructose generated power from aerobically cultured $S$. oneidensis. Power generation from these fuels was a result of the successful scrubbing of oxygen within the fuel cell chamber, which increases the power produced from a S. oneidensis culture containing elevated levels of oxygen. Strictly anaerobic or sediment-based fuel cells are limited in their potential applications because of how and where they must be deployed to operate. The next generation of MFCs will have to consider ways to account for oxygen exposure in the anode considering a MFC used for terrestrial applications (non-sediment) will not be able to maintain the anaerobic conditions solely being considered by the MFC community at large. Miniaturization of MFCs and the subsequent use of three-dimensional electrodes increases the power density (per volume), generates power in the presence of air, and (with the observations from this work) takes advantage of aerobically acclimated $S$. oneidensis to produce power from fuels not considered useful for anaerobic MFCs using the same bacteria.

\section{Acknowledgements}

This work was funded by the Office of Naval Research (NRL 6.2 Program Element Number 62123N). The authors want to acknowledge the mentorship program at Thomas Jefferson High School for Science and Technology (Annandale, VA) for providing summer research for Ms. Byrd. 


\section{Appendix A. Supplementary data}

Supplementary data associated with this article can be found, in the online version, at doi:10.1016/j.bios.2007.08.021.

\section{References}

Beliaev, A.S., Klingeman, D.M., Klappenbach, J.A., Wu, L., Romine, M.F., Tiedje, J.M., Nealson, K.H., Fredrickson, J.K., Zhou, J., 2005. J. Bacteriol. 187 (20), 7138-7145.

Beliaev, A.S., Thompson, D.K., Khare, T., Lim, H., Brandt, C.C., Li, G., Murray, A.E., Heidelberg, J.F., Giometti, C.S., Yates III, J., Nealson, K.H., Tiedje, J.M., Zhou, J., 2002. Omics 6 (1), 39-60.

Chaudhuri, S.K., Lovley, D.R., 2003. Nat. Biotechnol. 21 (10), 1229-1232.

Coates, J.D., Councell, T., Ellis, D.J., Lovley, D.R., 1998. Anaerobe 4 (6), 277-282.

Coates, J.D., Phillips, E.J.P., Lonergan, D.J., Jenter, H., Lovley, D.R., 1996. Appl. Environ. Microbiol. 62 (5), 1531-1536.

Giometti, C.S., Khare, T., Tollaksen, S.L., Tsapin, A., Zhu, W., Yates III, J.R., Nealson, K.H., 2003. Proteomics 3 (5), 777-785.

Halliwell, B., 2001. Mutat. Res. 475 (1,2), 29-35.

He, Z., Minteer, S.D., Angenent, L.T., 2005. Environ. Sci. Technol. 39 (14), 5262-5267.

Heidelberg, J.F., Paulsen, I.T., Nelson, K.E., Gaidos, E.J., Nelson, W.C., Read, T.D., Eisen, J.A., Seshadri, R., Ward, N., Methe, B., Clayton, R.A., Meyer, T., Tsapin, A., Scott, J., Beanan, M., Brinkac, L., Daugherty, S., DeBoy, R.T., Dodson, R.J., Durkin, A.S., Haft, D.H., Kolonay, J.F., Madupu, R., Peterson, J.D., Umayam, L.A., White, O., Wolf, A.M., Vamathevan, J., Weidman, J., Impraim, M., Lee, K., Berry, K., Lee, C., Mueller, J., Khouri, H., Gill, J., Utterback, T.R., McDonald, L.A., Feldblyum, T.V., Smith, H.O., Venter, J.C., Nealson, K.H., Fraser, C.M., 2002. Nat. Biotechnol. 20 (11), 1118-1123.

Kim, B.H., Ikeda, T., Park, H.S., Kim, H.J., Hyun, M.S., Kano, K., Takagi, K., Tatsumi, H., 1999. Biotechnol. Tech. 13 (7), 475-478.

Kim, H.J., Park, H.S., Hyun, M.S., Chang, I.S., Kim, M., Kim, B.H., 2002. Enzyme Microb. Technol. 30 (2), 145-152.

Kim, N., Choi, Y., Jung, S., Kim, S., 2000. Biotechnol. Bioeng. 70 (1), 109-114.

Kolker, E., Picone, A.F., Galperin, M.Y., Romine, M.F., Higdon, R., Makarova, K.S., Kolker, N., Anderson, G.A., Qiu, X., Auberry, K.J., Babnigg, G., Beliaev, A.S., Edlefsen, P., Elias, D.A., Gorby, Y.A., Holzman, T., Klappenbach, J.A., Konstantinidis, K.T., Land, M.L., Lipton, M.S., McCue, L.-A., Monroe, M., Pasa-Tolic, L., Pinchuk, G., Purvine, S., Serres, M.H., Tsapin, S., Zakrajsek, B.A., Zhu, W., Zhou, J., Larimer, F.W., Lawrence, C.E., Riley, M., Collart, F.R., Yates III, J.R., Smith, R.D., Giometti, C.S., Nealson, K.H., Fredrickson, J.K., Tiedje, J.M., 2005. Proc. Natl. Acad. Sci. U.S.A. 102 (6), 2099-2104.
Lee, S.A., Choi, Y., Jung, S., Kim, S., 2002. Bioelectrochemistry 57 (2), 173-178.

Lin, W.C., Coppi, M.V., Lovley, D.R., 2004. Appl. Environ. Microbiol. 70 (4), 2525-2528.

Liu, H., Ramnarayanan, R., Logan, B.E., 2004. Environ. Sci. Technol. 38 (7), 2281-2285.

Logan, B.E., Hamelers, B., Rozendal, R., Schroeder, U., Keller, J., Freguia, S., Aelterman, P., Verstraete, W., Rabaey, K., 2006. Environ. Sci. Technol. 40 (17), 5181-5192.

Lovley, D.R., 1987. Geomicrobiol. J. 5 (3/4), 375-399.

Lovley, D.R., 1993. Annu. Rev. Microbiol. 47, 263-290.

Lovley, D.R., 2000. Environ. Microbe Met. Interact., 3-30.

Lovley, D.R., 2006. Nat. Rev. Microbiol. 4 (7), 497-508.

Lovley, D.R., Coates, J.D., Saffarini, D.A., Lonergan, D.J., 1997. Transition Met. Microb. Metab., 187-215.

Lovley, D.R., Holmes, D.E., Nevin, K.P., 2004. Adv. Microb. Physiol. 49, 219-286.

Min, B., Logan, B.E., 2004. Environ. Sci. Technol. 38 (21), 5809-5814.

Nealson, K.H., Belz, A., McKee, B., 2002. Antonie Van Leeuwenhoek 81 (1-4), 215-222.

Nealson, K.H., Saffarini, D., 1994. Annu. Rev. Microbiol. 48, 311-343.

Niessen, J., Schroeder, U., Scholz, F., 2004. Electrochem. Commun. 6 (9), 955-958.

Park, D.H., Zeikus, J.G., 2003. Biotechnol. Bioeng. 81 (3), 348-355.

Pham, C.A., Jung, S.J., Phung, N.T., Lee, J., Chang, I.S., Kim, B.H., Yi, H., Chun, J., 2003. FEMS Microbiol. Lett. 223 (1), 129-134.

Rabaey, K., Verstraete, W., 2005. Trends Biotechnol. 23 (6), 291-298.

Ringeisen, B.R., Henderson, E., Wu, P.K., Pietron, J., Ray, R., Little, B., Biffinger, J.C., Jones-Meehan, J.M., 2006. Environ. Sci. Technol. 40 (8), 2629-2634.

Ringeisen, B.R., Ray, R., Little, B., 2007. J. Power Source 165, 591597.

Roncel, M., Ortega, J.M., Losada, M., 2001. Eur. J. Biochem. 268 (18), 4961-4968.

Saffarini, D.A., DiChristina, T.J., Bermudes, D., Nealson, K.H., 1994. FEMS Microbiol. Lett. 119 (3), 271-278.

Scott, J.H., Nealson, K.H., 1994. J. Bacteriol. 176 (11), 3408-3411.

Shukla, A.K., Suresh, P., Berchmans, S., Rajendran, A., 2004. Curr. Sci. 87 (4), 455-468.

Tender, L.M., Reimers, C.E., Stecher, H.A., Holmes, D.E., Bond, D.R., Lowy, D.A., Pilobello, K., Fertig, S.J., Lovley, D.R., 2002. Nat. Biotechnol. 20 (8), 821-825.

Yang, C., Rodionov, D.A., Li, X., Laikova, O.N., Gelfand, M.S., Zagnitko, O.P., Romine, M.F., Obraztsova, A.Y., Nealson, K.H., Osterman, A.L., 2006. J. Biol. Chem. 281 (40), 29872-29885.

Zuo, Y., Maness, P.-C., Logan, B.E., 2006. Energy Fuels 20 (4), 1716-1721. 